\title{
Detection and molecular characterization of cactus witches'- broom disease associated with a group 16Srll phytoplasma in northern areas of China
}

\author{
Zheng-Nan Li ${ }^{1}$, Lei Zhang ${ }^{2}$, Ping Liu ${ }^{1}$, Yao-Bo Bai ${ }^{1}$, Xiao-Gang Yang ${ }^{3} \&$ Yun-Feng Wu ${ }^{1}$ \\ ${ }^{1}$ State Key Laboratory of Crop Stress Biology in Arid Areas and Key Laboratory of Crop Pest Integrated Pest Management \\ on the Loess Plateau of Ministry of Agriculture, College of Plant Protection, Northwest A\&F University, Yangling, Shaanxi, \\ 712100, China; ${ }^{2}$ College of Forestry, Northwest A\&F University, Yangling, Shaanxi, 712100, China; ${ }^{3}$ College of Life Science \\ and Chemistry, Hulun Buir College, Inner Mongolia Hulun Buir, 0210083, China
}

Author for correspondence: Yun-Feng Wu, e-mail: wuyf@nwsuaf.edu.cn

\begin{abstract}
In July 2010, cacti with typical phytoplasma symptoms were observed in Yangling district, Shaanxi Province, China. Based on amplification of 16S rRNA gene, phytoplasmas were detected by polymerase chain reaction (PCR) driven with universal primer pairs P1/ P7, followed by a nested PCR with universal primer pairs R16F2nR16R2. Fragments of expected sizes (1.8 kb and $1.2 \mathrm{~kb})$ were obtained from symptomatic samples, but not from the asymptomatic samples. Sequencing results and BLASTn analysis of the $1806 \mathrm{bp}$ products (P1/P7) showed that the phytoplasma belonged to group 16SrII. Phylogenetic analysis to the R16F2nR16R2 region and virtual restriction fragment length polymorphism (RFLP) indicated that this phytoplasma was a member of subgroup 16SrII-C. This is the first reported occurrence of a peanut witches'-broom phytoplasma infecting plants in northern areas of China.
\end{abstract}

Key words: Opuntia spp, nested PCR, phytoplasma, RFLP in silico.

Cacti (Opuntia spp.) are perennial, evergreen, succulent plants native to arid areas of the Americas (Wei et al., 2007). On account of their artistic appearance and economic value, many cacti have been cultivated and introduced to other parts of the world as ornamentals, forages or fruits. Cacti are sensitive to phytoplasma infection and develop characterized symptoms such as witches'-broom, green-mosaic patterns in the epidermis (Cai et al., 2008), stunted growth and cladodes (Bertaccini et al., 2007). A series of reports related to phytoplasma infections of cacti come out from several countries including China, Mexico, Italy and Lebanon, and these phytoplasmas belong, respectively, to the peanut witches'-broom group (16SrII), the elm yellows group (16SrV) and the aster yellows group (16SrI) (Granata et al., 2006; Bertaccini et al., 2007; Cai et al., 2008; Zak et al., 2011).

In July 2010, cacti with phytoplasma-like symptoms of newborn fleshy stem gathered on the old ones (witches'-broom) and stunted growth were observed in Yangling district, Shaanxi Province, China (Figure 1). Fleshy stem samples were collected from five symptomatic and two asymptomatic plants, respectively. The common parthenium witches'-broom phytoplasma belonging to subgroup 16SrII-A was used as reference strain (Li et al., 2011).

Total DNA of the cacti stem sample was extracted using DNeasy Plant Mini kit (Qiagen) to avoid being affected by the high concentrations of polysaccharide, while the reference phytoplasma DNA was obtained by the cetyltrimethyl ammonium bromide (CTAB) method

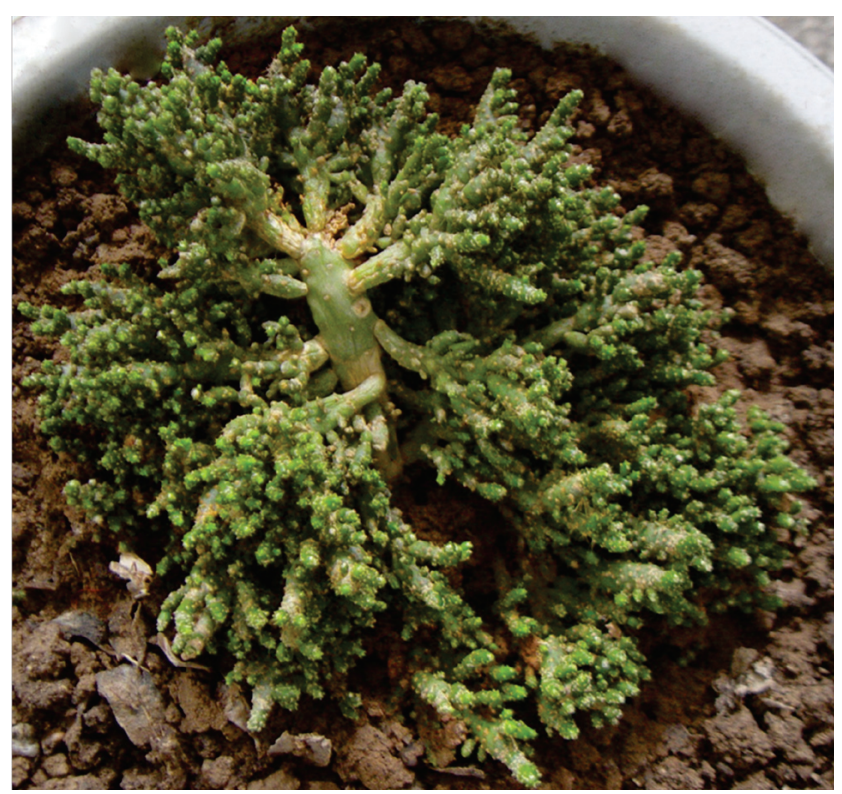

FIGURE 1 - Cactus with witches'-broom and stunted growth symptoms. 
(Kollar et al., 1990). Based on the total DNA template, first polymerase chain reaction (PCR) amplification was performed with phytoplasma universal primer pairs P1/P7 (Deng and Hiruki, 1991), followed by a nested PCR with universal primer pairs R16F2n/R16R2 (Gundersen and Lee 1996). The first PCR amplicons were purified, cloned into pMD18-T simple vector (TaKaRa biotechnology Co.Ltd, Dalian, China) and transformed into Escherichia coli strain JM109. Five positive clones were selected and sequenced by TaKaRa (Dalian, China).

The nucleotide sequence data were analyzed by the LaserGene program (version 7.1.0, DNASTAR, Madison, USA). National Center for Biotechnology Information's (NCBI) BLAST $n$ program (http://blast.ncbi.nlm.nih.gov/) was used to search the similar sequences from nucleotide sequence databases. DNAMAN software version 6.0 (Lynnon Biosoft, Canada) was employed to align the R16F2n/R16R2 region of the similar sequences and the target sequence one by one. A phylogenetic tree was constructed by the neighbour-joining method with a 1000replicate bootstrap search using MEGA4 (Tamura et al., 2007). Virtual RFLP analyses were performed by in silico digestion analysis of the R16F2n/R16R2 region of the $16 \mathrm{~S}$ rRNA gene sequences with the 17 restriction endonucleases previously designed for phytoplasma classification (Lee et al., 1993). Virtual RFLP pattern identification and similarity coefficient calculation were performed with a Perl program developed by Wei et al. (2008).

The expected sizes of $1.8 \mathrm{~kb}$ and $1.2 \mathrm{~kb}$ fragments were obtained from both symptomatic samples and positive controls, but not from the asymptomatic samples. The five selected clones produced identical fragments (1806 bp) and one sequence was deposited in GenBank (Accession No. JN582265). We denominated it as cactus witches'-broom phytoplasma strain Yangling (CaWBYL).

BLAST $n$ analysis retrieved 100 nucleotide sequences with high identity to JN582265. These 100 sequences were from phytoplasma 16S rRNA gene sequences related to the peanut witches'-broom group, and 30 of them were from cactus witches'-broom phytoplasmas. The CaWB-YL sequence (JN582265) showed the lowest identity, of $96.79 \%$, with AY 169322 (alfalfa witches'-broom phytoplasma) and the same highest identity $(99.92 \%)$ with 16 sequences which include 10 cactus witches'-broom phytoplasma sequences (EU099561, EU099560, EU099554, EU099553, EU099565, EU099559, EU099550, EU099564, EU099562, EU099557); for others, the identity with CaWBYL ranged from $96.87 \%$ to $99.84 \%$.

The phylogenetic tree based on the CaWB-YL R16F2n/R16R2 region of 16S rRNA and those from 25 other phytoplasmas shows that CaWB-YL and the eleven 16SrII group phytoplasmas are in the same branch. Furthermore, within this branch, CaWB-YL clusters with member of subgroup 16SrII-C (Figure 2). The virtual 16S rRNA RFLP patterns of CaWB-YL were identical to those from representative strains of the 16SrII-C subgroup (Figure 3). The pairwise similarity coefficients between CaWB-YL and representative strains of 16SrII subgroups were above 0.92 , and the cactus witches'broom phytoplasma isolate YNO1 (AJ293216), a member of 16SrII-C, has the highest one (1.0) with CaWB-YL (Table 1). These results confirm that CaWB-YL is a member of subgroup $16 \mathrm{Sr}$ II-C.

In recent years, more than 40 phytoplasma-associated diseases have been reported in China according to the Science Citation Index (SCI) (Figure 4). Phytoplasmas of groups $16 \mathrm{Sr}-\mathrm{I}$, $-\mathrm{II}$ and $-\mathrm{V}$ seem to be the most important

TABLE 1 - Similarity coefficients derived from analysis of virtual RFLP patterns of 16S rDNA R16F2n/R16R2 region from CaWB-YL and representative strains in $16 \mathrm{SrII}$ subgroups $(\mathrm{A} / \mathrm{B} / \mathrm{C} / \mathrm{D} / \mathrm{E} / \mathrm{F} / \mathrm{G} / \mathrm{H} / \mathrm{I} / \mathrm{J} / \mathrm{K} / \mathrm{L})$

\begin{tabular}{|c|c|c|c|c|c|c|c|c|c|c|c|c|c|}
\hline Serial & Strain/ Accession no. & 1 & 2 & 3 & 4 & 5 & 6 & 7 & 8 & 9 & 10 & 11 & 12 \\
\hline 1 & II-A/L33765 & 1.00 & & & & & & & & & & & \\
\hline 2 & II-B/U15442 & 0.88 & 1.00 & & & & & & & & & & \\
\hline 3 & II-C/AJ293216 & 0.94 & 0.94 & 1.00 & & & & & & & & & \\
\hline 4 & II-D/Y10097 & 0.95 & 0.91 & 0.97 & 1.00 & & & & & & & & \\
\hline 5 & II-F/EU099556 & 0.91 & 0.93 & 0.97 & 0.94 & 1.00 & & & & & & & \\
\hline 6 & II-G/EU099568 & 0.91 & 0.91 & 0.97 & 0.94 & 0.94 & 1.00 & & & & & & \\
\hline 7 & II-H/EU099569 & 0.90 & 0.92 & 0.96 & 0.93 & 0.93 & 0.95 & 1.00 & & & & & \\
\hline 8 & II-I/EU099551 & 0.90 & 0.89 & 0.95 & 0.92 & 0.92 & 0.92 & 0.91 & 1.00 & & & & \\
\hline 9 & II-J/EU099552 & 0.88 & 0.88 & 0.94 & 0.91 & 0.91 & 0.91 & 0.90 & 0.89 & 1.00 & & & \\
\hline 10 & II-K/EU099572 & 0.88 & 0.88 & 0.94 & 0.91 & 0.91 & 0.91 & 0.90 & 0.89 & 0.88 & 1.00 & & \\
\hline 11 & II-L/EU099546 & 0.87 & 0.87 & 0.92 & 0.90 & 0.89 & 0.89 & 0.88 & 0.88 & 0.87 & 0.87 & 1.00 & \\
\hline 12 & CaWB-YL/JN582265 & 0.94 & 0.94 & 1.00 & 0.97 & 0.97 & 0.97 & 0.96 & 0.95 & 0.94 & 0.94 & 0.92 & 1.00 \\
\hline
\end{tabular}




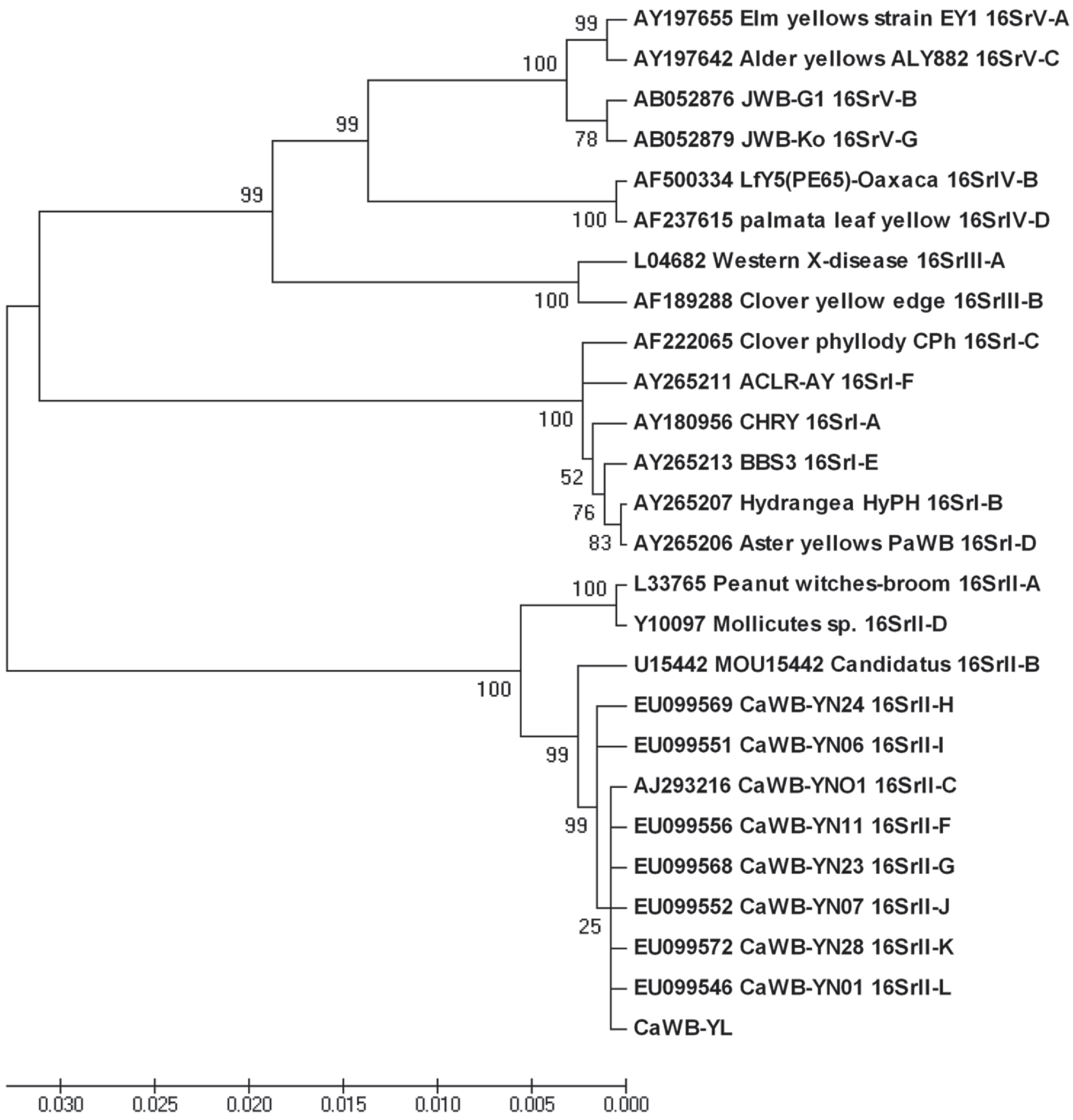

FIGURE 2 - Phylogenetic tree constructed based on the R16F2n/R16R2 region of 16S rRNA genes of CaWB-YL and 25 other phytoplasmas by the neighbor-joining method. Numbers on branches indicate the bootstrap value (expressed as percentages of 1,000 replications).

ones, considering the high number of diseases they cause. Phytoplasmas of groups $16 \mathrm{Sr}-\mathrm{I}$ and $-\mathrm{V}$, however, are distributed more widely than the ones of group 16SrII; the former occurred in both northern and southern areas of China, while the latter were reported only in Hainan and Yunnan provinces, which are located in southern areas of the country (Figure 4). The climate of these two southern provinces is tropical and subtropical, and the presence of phytoplasmas of group 16SrII is not unusual, for these phytoplasmas mostly occur in tropical and subtropical regions. But in the present study, a group 16SrII phytoplasma was found in northern Shaanxi province, which has a temperate climate. This could mean that group 16SrII phytoplasmas are moving northward in China. It may be because of the flower trade between northern and southern areas of China, or the vector has spread due to climate change. In fact, there 


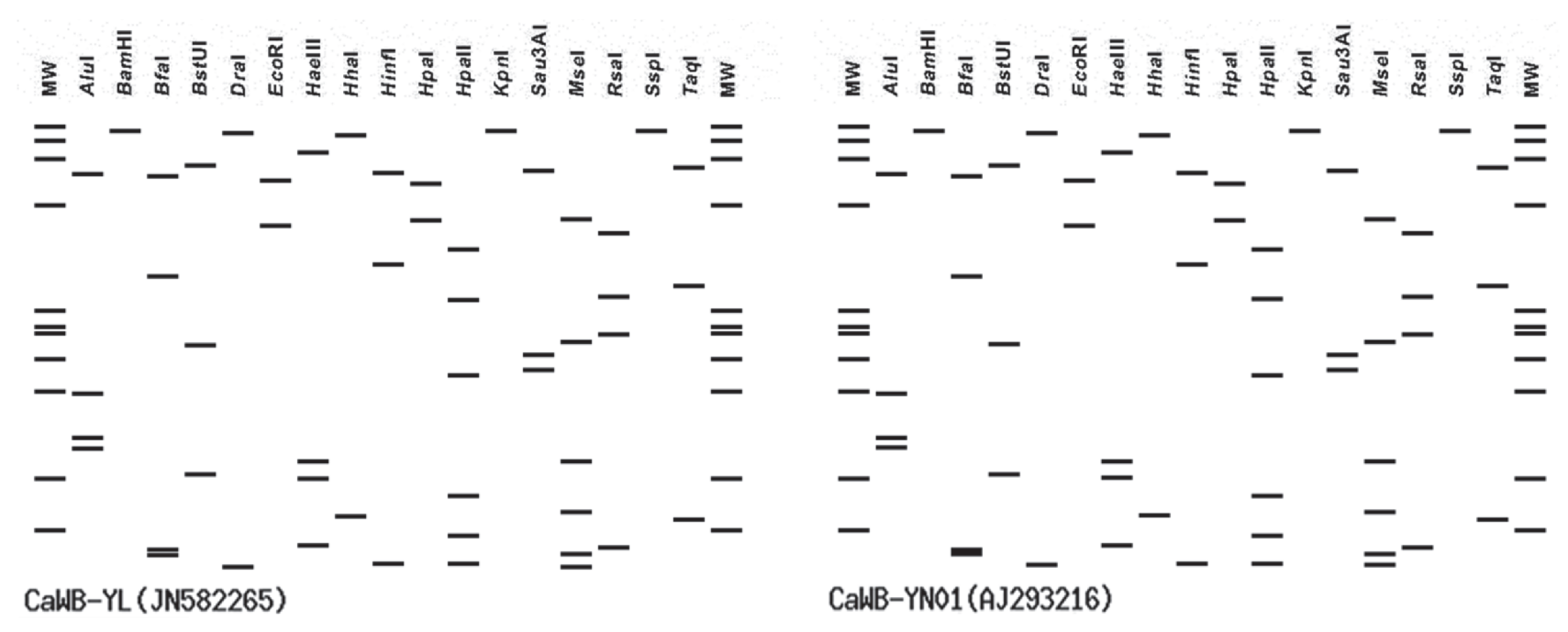

FIGURE 3 - Virtual RFLP patterns derived from in silico digestions of R16F2n/R16R2 region of phytoplasmas using 17 restriction enzymes: Alu I, BamH I, Bfa I, BstU I (Tha I), Dra I, EcoR I, Hae III, Hha I, Hinf I, Hpa I, Hpa II, Kpn I, Aau3AI (Mbo I), Mse I, Rsa I, Ssp I and Taq I. Left: RFLP profiles from CaWB-YL (JN582265); Right: RFLP profiles from CaWB-YNO1 (AJ293216).

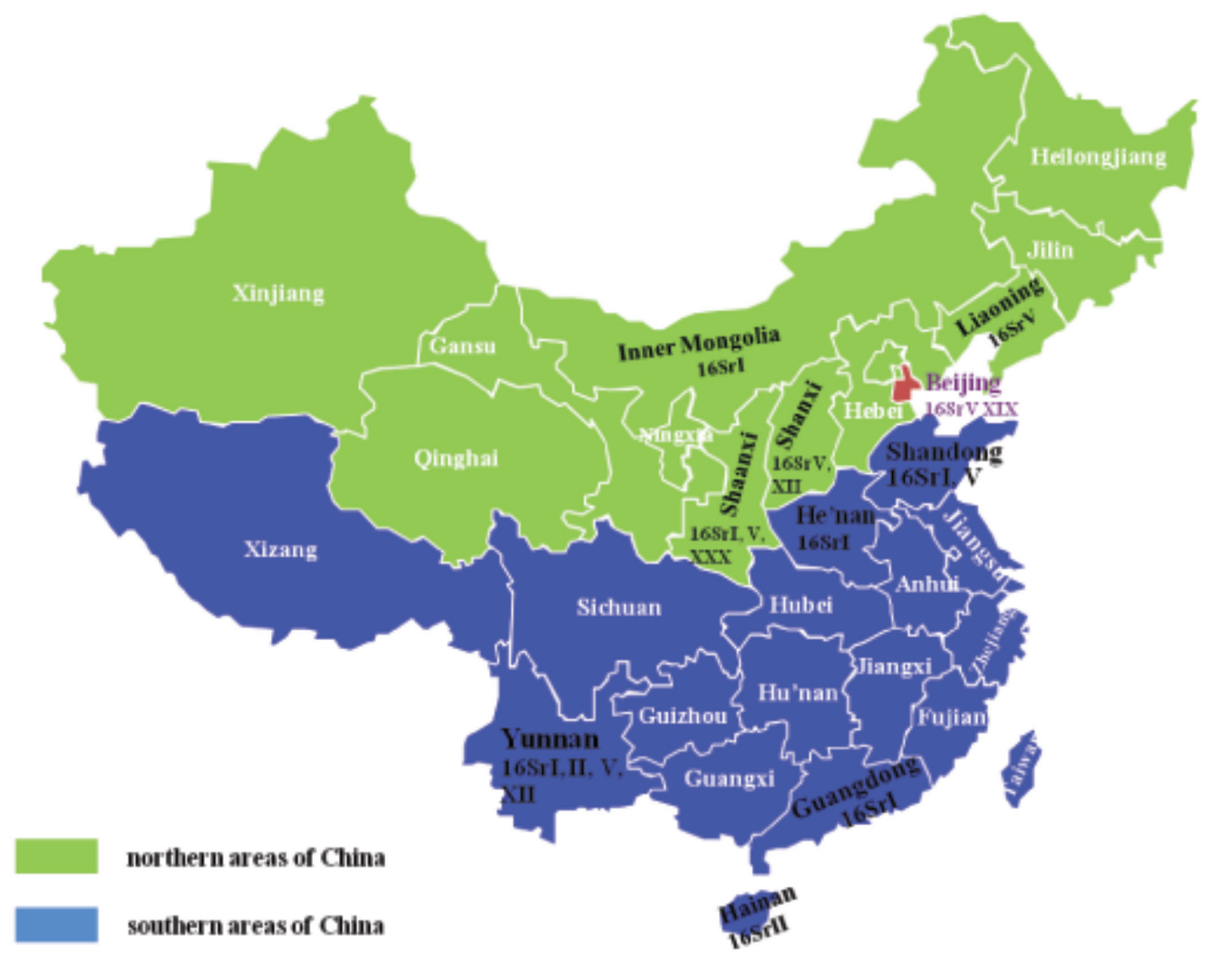

FIGURE 4 - Map of China showing the geographical distribution of phytoplasmas from each 16S rRNA gene RFLP group in the country.

is a similar report on the spread of tomato yellow leaf curl virus into temperate areas in China (Zhang et al., 2009), in which climate change was also proposed as one possible explanation. Although the exact reasons are not yet clear, the finding of phytoplasma of group 16SrII in northern, temperate areas of China is a significant event.

\section{ACKNOWLEDGEMENTS}

This research was supported by the 111 project from the Education Ministry of China, (No.B07049), and the National Natural Science Foundation of China (30970133). 


\section{REFERENCES}

Bertaccini A, Calari A, Felker P (2007) Developing a method for phytoplasma identification in cactus pear samples from California. Bulletin of Insectology 60:257-258.

Cai H, Wei W, Davis RE, Chen H, Zhao Y (2008) Genetic diversity among phytoplasmas infecting Opuntia species: Virtual RFLP analysis identifies new subgroups in the peanut witches'broom phytoplasma group. International Journal of Systematic and Evolutionary Microbiology 58:1448-1457.

Deng S, Hiruki C (1991) Amplification of 16S rRNA genes from culturable and nonculturable mollicutes. Journal of Microbiological Methods 14:53-61.

Granata G, Paltrinieri S, Botti S, Bertaccini A (2006) Aetiology of Opuntia ficus-indica malformations and stunting disease. Annals of Applied Biology 149:317-325.

Gundersen DE, Lee IM (1996) Ultrasensitive detection of phytoplasmas by nested-PCR assays using two universal primer pairs. Phytopathologia Mediterranea 35:144-151.

Kollar A, Seemüller E, Bonnet F, Saillard C, Bové JM (1990) Isolation of the DNA of various plant pathogenic mycoplasmalike organisms from infected plants. Phytopathology 80:233-237.

Lee IM, Hammond RW, Davis RE, Gundersen DE (1993) Universal amplification and analysis of pathogen 16S rDNA for classification and identification of mycoplasma-like organisms. Phytopathology 83:834-842.

Li Z, Zhang L, Che H, Liu H, Chi M, Luo D, Li Y, Chen W, Wu Y (2011) A disease associated with phytoplasma in Parthenium hysterophorus. Phytoparasitica 39:407-410.

Tamura K, Dudley J, Nei M, Kumar S (2007) MEGA4: Molecular evolutionary genetics analysis (MEGA) software version 4.0. Molecular Biology and Evolution 24:1596-1599.

Wei W, Cai H, Chen H, Davis RE, Zhao Y (2007) First report of a natural infection of Opuntia sp. by a 'Candidatus Phytoplasma asteris'-related phytoplasma in China. Plant Disease 91:461.

Wei W, Lee IM, Davis RE, Suo X, Zhao Y (2008) Automated RFLP pattern comparison and similarity coefficient calculation for rapid delineation of new and distinct phytoplasma $16 \mathrm{Sr}$ subgroup lineages. International Journal of Systematic and Evolutionary Microbiology 58:2368-2377.

Zak LF, Yáñz-Morales MJ, Alanis-Martínez I, González-Pérez E (2011) New hosts of 16SrI phytoplasma group associated with edible Opuntia ficus-indica crop and its pests in Mexico. African Journal of Microbiology Research 5:910-918.

Zhang H, Gong H, Zhou X (2009) Molecular characterization and pathogenicity of tomato yellow leaf curl virus in China. Virus Genes 39:249-255.

TPP 405 - Received 17 September 2011 - Accepted 5 April 2012 Section Editor: F. Murilo Zerbini 